\title{
On Conformally Coupled General Relativity
}

\author{
Andrej Arbuzov ${ }^{1,2, *}$ and Boris Latosh ${ }^{2,3, * *}$ \\ ${ }^{1}$ Bogoliubov Laboratory for Theoretical Physics, JINR, Dubna 141980, Russia \\ ${ }^{2}$ Dubna State University, Universitetskaya str. 19, Dubna 141982, Russia \\ ${ }^{3}$ Department of Physics and Astronomy, University of Sussex, Brighton, BN1 9QH, United Kingdom
}

\begin{abstract}
A gravity model based on the conformal symmetry is presented. To specify the structure of the general coordinate transformations the Ogievetsky theorem is applied. The nonlinear symmetry realization approach is used. Canonical quantization is performed with the use of reparameterizationinvariant time and the Arnowitt-Deser-Misner foliation. Renormalizability of the constructed quantum gravity model is discussed.
\end{abstract}

\section{Introduction}

General Relativity (GR) is the commonly accepted theory of spacetime. It is verified by many experimental and observational tests at different scales $[1,2]$. The recent discovery of gravitational waves provided further support of the GR viability at the classical level [3]. Nevertheless, the classical general relativity is unable to provide a reasonable description of gravitational phenomena at high energies and should be either quantized or replaced by a new theory, see e.g. review [4].

In this report, we revisit a specific approach to quantum gravity, which we call Conformally Coupled General Relativity (CCGR) [5]. The approach involves a series of techniques: nonlinear symmetry realizations [6-8] which are applied to implement the Ogievetsky theorem [9]; the Hamiltonian formalism [10, 11]; the conformal coupling of matter to gravity [12-14]; and the parameterization-invariant time [15-17]. This approach was developed in the series of papers [18-20]. Our main aim here is to present it in a self-consistent comprehensive form. The main focus of this paper is the quantum feature of CCGR, since in previous papers the latter was discussed very briefly and obscure.

Using the Ogievetsky theorem [9] for gravity quantization is very promissing since it specifies the structure of general coordinate transformations. The theorem states that every generator of the general coordinate transformations is a linear combination of commutators formed by generators from the special linear $S L(4, \mathbb{R})$ and conformal $C(1,3)$ groups. Thus, the coordinate transformations can be expressed with a finite number of generators. How to construct a theory of gravity with these groups was suggested by Borisov and Ogievetsky [21]. Their idea was to use the formalism of nonlinear symmetry realizations [6-8]. Despite the fact that $C(1,3)$ and $S L(4, \mathbb{R})$ symmetries are assumed to be fundamental, only the Lorentz subgroup receives a linear realization. Thus the model receives the standard

\footnotetext{
*e-mail: arbuzov@theor.jinr.ru

**e-mail: latosh.boris@gmail.com
} 
Lorentzian currents, but it has no conservation laws for the rest of the symmetry. The vierbein (tetrad) formalism was used to construct the group representation.

The benefits of this method appear at the level of quantum theory, as it introduces a new symmetry which imposes additional constrains and dictates the choice of dynamical varibales. As we will see below, the (nonlinear) structure of GR is considerably simplified for the properly chosen set of variables.

Another important issue is how to define the coupling of gravity to matter. The coupling should be driven by some fundamental physical principle, in order to narrow down the number of possibilities. Application of the conformal symmetry principle for this purpose was suggested by Dirac and Deser [12-14]. Their approach can be used in the following way. First, one constructs a coupling of matter to gravity in a conformally-invariant way. Second, following Deser, one separates the conformal metric at the level of an action via conformal transformations. Thus, the action turns out to be separated into two parts: one drives the cosmological evolution and the other one drives local gravitational interactions. To the best of our knowledge, Penrose, Chernikov and Tagirov [22, 23] were the first who wrote the Hilbert action in such a form. Usage of the conformal gravity coupling gave birth to the conformal cosmological model [24-27].

For quantization we will apply the canonical Hamiltonian approach formulated by Arnowitt, Deser and Misner (ADM) [10] and founded by Dirac [11]. It treats a fourdimensional spacetime as a series of three-surfaces with dynamical geometry. The implication of the ADM foliation gives rise to the time reparameterization problem that was addressed in refs. $[16,17,28]$. Within quantum theory, time is treated as an external parameter mapping the system's evolution. In the realm of gravity models, time is merely a coordinate that can be chosen arbitrarily. But in the realm of cosmology, one expects to associate time with a cosmological epoch and thus related it to the cosmological scale factor in accord with the Einstein cosmological principle [29].

\section{Conformally Coupled General Relativity}

Let us separate the conformal degrees of freedom from the full metric in the standard way:

$$
g_{\mu \nu} d x^{\mu} \otimes d x^{\nu}=e^{-2 D} \widetilde{g}_{\mu \nu} d \chi^{\mu} \otimes d \chi^{\nu} .
$$

Here, $g_{\mu \nu}$ in the full (Einstein) metric; $\widetilde{g}_{\mu \nu}$ in the conformal metric; $x_{\mu}$ are arbitrary coordinates on a spacetime; $\chi_{\mu}$ are the correspondent conformally-invariant coordinates; and $D$ is the conformal degree of freedom, also known as the dilaton. The metric parameterization (1) results in the Penrose-Chernikov-Tagirov action [22, 23]. At this level, we impose the conformal coupling to matter and obtain the following action:

$$
\begin{gathered}
S_{\text {Hilbert }}=\int d^{4} x \sqrt{-g}\left[\frac{M_{P}^{2}}{16 \pi}(R-2 \Lambda)+L_{\text {matter }}\left(g_{\mu \nu}\right)\right] \rightarrow \\
S_{\mathrm{CCGR}}=\int d^{4} \chi \sqrt{-\widetilde{g}}\left[\frac{\widetilde{M}_{P}^{2}}{16 \pi}(\widetilde{R}-2 \widetilde{\Lambda})+\frac{3 \widetilde{M}_{P}^{2}}{8 \pi}\left(\widetilde{g}^{\mu \nu} \nabla_{\mu} D \nabla_{\nu} D\right)+L_{\text {matter }}\left(\widetilde{g}_{\mu \nu}\right)\right] .
\end{gathered}
$$

Here, $\Lambda$ is the cosmological constant, $\widetilde{\Lambda}=e^{-2 D} \Lambda$ is the conformal cosmological constant, $M_{P}$ is the Planck mass, and $\widetilde{M}_{P}=M_{P} e^{-D}$ is the conformal Planck mass. Action (3) is not fully equivalent to the Hilbert action (2) and does not reproduce general relativity in all detail. The key point is in the conformal gravitational coupling. The Hilbert action (2) describes matter coupled to the full (Einstein) metric, which does not admit conformal invariance, while the CCGR action (3) preserves the coupling invariant under conformal transformations. In such 
a way, at this level, we do not consider general relativity, but its modification. We introduce the cosmological constant in the model for the sake of generality and treat it as a free model parameter. However, verification by Type Ia supernovae data showed that the conformal cosmological model based on the action (3) provides a good fit for cosmological data with a vanishing cosmological constant [27]. That model is an alternative to the standard $\Lambda \mathrm{CDM}$ cosmological model.

We define the (conformal) tetrad (vierbein) in the following way:

$$
g_{\mu \nu} d x^{\mu} \otimes d x^{v}=e^{-2 D} \widetilde{g}_{\mu \nu} d \chi^{\mu} \otimes d \chi^{v}=e^{-2 D} \eta_{(\mu)(v)} \omega_{(\mu)} \otimes \omega_{(v)},
$$

where $\omega_{(\mu)}$ is the (conformal) tetrad basis subjected to the nonlinear symmetry realization.

Due to the conformal invariance, any conformal transformation $\widetilde{g}_{\mu \nu} \rightarrow e^{2 \Omega} \widetilde{g}_{\mu \nu}$ are supplied with a dilaton transformation $D \rightarrow D+\Omega$ preserving the form of the metric (1). In terms of the (conformal) tetrad, the conformal symmetry is given by

$$
\left\{\begin{array}{l}
\omega_{(\mu)} \rightarrow e^{\Omega} \omega_{(\mu)} \\
D \rightarrow D+\Omega
\end{array}\right.
$$

The conformal metric components and the dilaton are dependent variables, because we did not introduce new degrees of freedom in transition from (2) to (3). We relate the dilaton to the three-metric ${ }^{(3)} g_{m n}$ defined by the full metric $g_{\mu \nu}$ as

$$
D=-\frac{1}{6} \ln \operatorname{det}^{(3)} g_{m n}
$$

One cannot express the dilaton directly through the conformal metric because of the symmetry (5). Instead, one can fix the gauge of variables $\widetilde{g}$ and $D$ in the full analogy with the gauge field theory.

In the refs. $[16,17]$, it was proposed to associate the physical time with the zero dilaton mode following the Einstein cosmological principle [29]. One can define the zero dilaton mode $\langle D\rangle$ which corresponds to its global (average) structure, and the fluctuation mode $\bar{D}$ which corresponds to the local dynamics:

$$
D\left(\chi^{0}, \chi^{1}, \chi^{2}, \chi^{3}\right)=\langle D\rangle\left(\chi^{0}\right)+\bar{D}\left(\chi^{0}, \chi^{1}, \chi^{2}, \chi^{3}\right) .
$$

This allows one to exclude all interferences between $\bar{D}$ and $\langle D\rangle$ from action (3) and to reduce it to the following form:

$$
\begin{aligned}
S & =\int d^{4} \chi \sqrt{\gamma} N\left[\frac{\widetilde{M}_{P}^{2}}{16 \pi}(\widetilde{R}-2 \widetilde{\Lambda})-\frac{3 \widetilde{M}_{P}^{2}}{8 \pi} \frac{1}{N^{2}}\left(\frac{\partial}{\partial \chi^{0}}\langle D\rangle\right)^{2}\right. \\
& \left.+\frac{3 \widetilde{M}_{P}^{2}}{8 \pi}\left(\widetilde{g}^{\mu \nu} \nabla_{\mu} \bar{D} \nabla_{v} \bar{D}\right)+L_{\text {matter }}\left(\widetilde{g}_{\mu \nu}\right)\right] .
\end{aligned}
$$

It gives a way to introduce the parameterization-invariant time. In full analogy with the dilaton, one separates the local and global parts of the lapse function $\mathrm{N}$ :

$$
N\left(\chi^{0}, \chi^{1}, \chi^{2}, \chi^{3}\right)=N_{0}\left(\chi^{0}\right) \mathcal{N}\left(\chi^{0}, \chi^{1}, \chi^{2}, \chi^{3}\right), \quad \frac{1}{N_{0}}=\left\langle\frac{1}{N}\right\rangle
$$

and $\mathcal{N}$ is subjected to the orthogonality condition: $\left\langle\mathcal{N}^{-1}\right\rangle=1$. The pure dilaton action turns out to be defined in terms of global components only:

$$
S_{\langle D\rangle}=-V_{0} \frac{3 \widetilde{M}_{P}^{2}}{8 \pi} \int d \chi^{0} N_{0}\left(\frac{1}{N_{0}} \frac{\partial}{\partial \chi^{0}}\langle D\rangle\right)^{2}
$$


Now any time reparameterization $\chi^{0} \rightarrow \chi^{\prime 0}$ can be absorbed into the definition of $N_{0}$. In this way, one can define the parameterization-invariant time $t$ as

$$
d t=N_{0} d \chi^{0} .
$$

The four-volume element can be cast in the form

$$
d^{4} x \sqrt{-\widetilde{g}}=d \chi^{0} N_{0} d^{3} \chi \sqrt{\gamma} \mathcal{N}=d t d^{3} \chi \sqrt{\gamma} \mathcal{N} .
$$

The ADM foliation admits the following decomposition of the CCGR action (8):

$$
\begin{aligned}
& S=S_{\text {Universe }}+S_{\text {Gravitons }}+S_{\text {Potential }}, \\
& S_{\text {Universe }}=-V_{0} \int d \chi^{0} N_{0} \frac{3 \widetilde{M}_{P}^{2}}{8 \pi}\left(\frac{1}{N_{0}} \frac{\partial}{\partial \chi^{0}}\langle D\rangle\right)^{2}, \\
& S_{\text {Gravitons }}=\int d \chi^{0} N_{0} \int d^{3} \chi \sqrt{\gamma} \mathcal{N} \frac{\widetilde{M}_{P}^{2}}{16 \pi}(\widetilde{R}-2 \widetilde{\Lambda}), \\
& S_{\text {Potential }}=\int d \chi^{0} N_{0} \int d^{3} \chi \sqrt{\gamma} \mathcal{N} \frac{3 \widetilde{M}_{P}^{2}}{8 \pi} \gamma^{a b} \partial_{a} \bar{D} \partial_{b} \bar{D} .
\end{aligned}
$$

The decomposition allows us to explore new features of CCGR. The decomposition was obtained due to the implementation of the new symmetry and introduction of the conformallyinvariant time coordinate $\langle D\rangle$. It shows that the cosmological evolution is driven by the mean (global) structure of the gravitational field described by the $\langle D\rangle$, while the local gravitational interaction is driven by the fluctuation part of the gravitational field component $\bar{D}$. In other words, we established independent parameterization of the global (cosmological) and local dynamics of the gravitation field. Action (13) is an explicit representation of this result. Local and global degrees of freedom interact with each other in a non-trivial way, and the interaction is given by (16). Corollaries of that model that take place in the classical regime were studied in refs. [18-20]. A detailed discussion of its cosmological implication, including the perturbation theory, is presented in ref. [34].

\section{Quantum Features of CCGR}

In this section, we demonstrate that one can construct a consistent approach to quantum CCGR in the low-energy regime. Such an approach was discussed briefly and obscure in previous papers [18-20]. Let us look for a solution of the classical field equations describing a solitary (nonlinear) plain wave propagating in the conformal space and associate it with a single graviton state in the quasi-classical regime. Then we will decompose the gravitational field into a series of (nonlinear) plain waves and obtains a quasi-classical graviton Lagrangian.

Note that this approach cannot be realized in general relativity because of the following. Within GR, one treats the metric as a dynamical variable, and it enters the Lagrangian in a nonlinear way. Therefore, one needs to associate quasi-classical gravitons with metric perturbations with nonlinear dynamics. In CCGR, one must use the tetrads and forms $\omega^{R}$ as dynamical variables carrying nonlinear symmetry realization. Note that this choice of variables is dicated by the assumed underlying (fundamental) symmetry. Forms $\omega^{R}$, unlike the metric, enter the gravity Lagrangian in a bilinear way due to the symmetry properties, thereby allowing one to avoid nonlinear dynamics. In other words, nonlinear representation of the new symmetry allows one (at least in the quasi-classical regime) to choose basic states with bilinear dynamics. 
To implement this program, one need first to choose the proper background for perturbation theory. Since we connected time with the cosmological epoch, we can only adopt the Friedman-Robertson-Walker (FRW) spacetime. In contrast to the standard approach, we do not consider the scale factor to be a classical (non-quantum) well-defined function, but expect it to be defined by dynamical quantum perturbations. The mean part of the dilaton $\langle D\rangle$ is related to the scale factor $a$ in the following simple way:

$$
\langle D\rangle=-\ln a .
$$

The conformal metric and the dilaton are dependent variables connected by the symmetry (5), so one can fix the gauge of the conformal metric by choosing $\gamma=1$ where $\gamma$ is the conformal three-metric determinant. This gauge is known as the Lichnerowicz gauge $[35,36]$.

Within the ADM foliation, action (15) reads (see ref. [5] for notations):

$$
S_{\text {Gravitons }}=\int d \chi^{0} N_{0} \int d^{3} \chi \mathcal{N}\left[G^{a b c d} K_{a b} K_{c d}+\sqrt{\gamma} \widetilde{R}^{(3)}\right],
$$

where $K_{a b}$ is the external curvature which is linear with respect to form $\omega^{R}$ :

$$
K_{a b}=\frac{1}{N}\left[\omega_{(m)(n)}^{R}\left(\partial_{t}\right) \omega_{(m) a} \omega_{(n) b}-\frac{1}{2}\left(\nabla_{a} N_{b}+\nabla_{b} N_{a}\right)\right] .
$$

We choose the following metric ansatz for a nonlinear plain wave solution:

$$
g=-d \chi^{0} \otimes d \chi^{0}+d \chi^{3} \otimes d \chi^{3}+e^{\Sigma}\left[e^{\sigma} d \chi^{1} \otimes d \chi^{1}+e^{-\sigma} d \chi^{2} \otimes d \chi^{2}\right]
$$

where $\sigma$ depends on $\chi^{3}$ and $\chi^{0}$, while $\Sigma$ depends only on $\chi^{1}$ and $\chi^{2}$. In this ansatz unlike in the standard metric variables, form $\omega^{R}$ admits the Taylor series expansion

$$
\omega_{(a)(b)}^{R}\left(\partial_{(c)}\right)=\int \frac{d^{3} k}{(2 \pi)^{3}} \frac{1}{\sqrt{2 \omega_{k}}} i k_{(c)}\left[\epsilon_{(a)(b)}^{R}(k) g_{k}^{+} e^{i k \cdot x}+\epsilon_{(a)(b)}^{R}(-k) g_{k}^{-} e^{-i k \cdot x}\right],
$$

where all scalar products are evaluated on the dreibein $\left(x \cdot k=k_{(a)} x_{(a)}\right)$. Functions $\epsilon_{(a)(b)}^{R}(k)$ correspond to from $\omega^{R}$ evaluated on a solitary nonlinear plain wave with wave vector $k$; and functions $g_{k}^{ \pm}$play the role of Fourier coefficients. In this way, formula (21) allows one to expand an arbitrary classical gravitational field in a series of nonlinear plain waves. Similar to weak waves in general relativity, $\epsilon_{(a)(b)}(k)$ is constrained by the following identities:

$$
\epsilon_{(a)(a)}^{R}(k)=0, \quad k_{(a)} \epsilon_{(a)(b)}^{R}(k)=0 .
$$

A single nonlinear wave fixes gravitons on the zero-mass shell $k_{\mu} k^{\mu}=0$ in full agreement with the classical theory.

\section{Conclusions}

In this way, we found a classical CCGR solution which describes nonlinear plain waves. The CCGR dynamical variables $\omega^{R}$ admit expansion in Fourier series with respect to the solitary nonlinear plain waves (21). Thus, one can associate the plain wave solution with a single graviton state, and the series (21) can be treated as an expansion in single graviton states. Action (15) defines the bilinear graviton dynamics, while the actions (14) and (16) define the cosmological dynamics and the gravity self-interaction, respectively. Therefore, we obtained a new way to perform perturbative quantization of gravity based on the new symmetries. The same method cannot be applied in the conventional general relativity, because it uses a different set of dynamical variables. 
Since the graviton action is bilinear in the dynamical variables, the resulting model appears to be trivial, i.e. it resembles QED since it does not contain graviton-graviton interactions. On the other hand, interactions of gravitons with the Standard Model fields are fully fixed by the conformal symmetry. Verification of the model renormalizability is straightforward. Note that due to the conformal symmetry the model is free from dimensionful coupling constants.

\section{References}

[1] C.M. Will, Living Rev. Rel. 17, 4 (2014)

[2] P.A.R. Ade et al. [Planck Collaboration], Astron. Astrophys. 594, A13 (2016)

[3] B.P. Abbott et al. [LIGO Scientific and VIRGO Collaborations], Phys. Rev. Lett. 118, no. 22, 221101 (2017)

[4] S. Nojiri, S. D. Odintsov and V. K. Oikonomou, Phys. Rept. 692, 1 (2017)

[5] A. Arbuzov and B. Latosh, Universe 4, no. 2, 38 (2018)

[6] S.R. Coleman, J. Wess and B. Zumino, Phys. Rev. 177, 2239 (1969)

[7] C.G. Callan, Jr., S. R. Coleman, J. Wess and B. Zumino, Phys. Rev. 177, 2247 (1969)

[8] D.V. Volkov, Fiz. Elem. Chast. Atom. Yadra 4, 3 (1973)

[9] V.I. Ogievetsky, Lett. Nuovo Cim. 8, 988 (1973)

[10] R.L. Arnowitt, S. Deser and C.W. Misner, Gen. Rel. Grav. 40, 1997 (2008)

[11] P.A.M. Dirac, Proc. Roy. Soc. Lond. A 246, 333 (1958)

[12] P.A.M. Dirac, Naturwiss. 60, 529 (1973)

[13] P.A.M. Dirac, Proc. Roy. Soc. Lond. A 333, 403 (1973)

[14] S. Deser, Annals Phys. 59, 248 (1970)

[15] P.A.M. Dirac, Can. J. Math. 2, 129 (1950)

[16] A.M. Khvedelidze, V.V. Papoian, Y.G. Palii and V.N. Pervushin, Phys. Lett. B 402, 263 (1997)

[17] V.N. Pervushin and V.I. Smirichinski, J. Phys. A 32, 6191 (1999)

[18] A.B. Arbuzov, B.M. Barbashov, A. Borowiec et al., Grav. Cosmol. 15, 199 (2009)

[19] A.B. Arbuzov, B.M. Barbashov, R.G. Nazmitdinov et al., Phys. Lett. B 691, 230 (2010)

[20] V.N. Pervushin, A.B. Arbuzov, B.M. Barbashov et al., Gen. Rel. Grav. 44, 2745 (2012)

[21] A.B. Borisov and V.I. Ogievetsky, Theor. Math. Phys. 21, 1179 (1975)

[22] R. Penrose, Relativity, Groups and Topology (Gordon and Breach, New York, 1964) 1.

[23] N.A. Chernikov and E.A. Tagirov, Ann. Inst. H. Poincare Phys. Theor. A 9, 109 (1968)

[24] D. Behnke, D.B. Blaschke, V.N. Pervushin and D. Proskurin, Phys. Lett. B 530, 20 (2002)

[25] D.B. Blaschke, S.I. Vinitsky, A.A. Gusev et al., Phys. Atom. Nucl. 67, 1050 (2004)

[26] B.M. Barbashov, V.N. Pervushin, A.F. Zakharov, V.A. Zinchuk, Int. J. Mod. Phys. A 21 , 5957 (2006)

[27] A.F. Zakharov and V.N. Pervushin, Int. J. Mod. Phys. D 19, 1875 (2010)

[28] A.B. Arbuzov and A.E. Pavlov, arXiv:1710.01528 [gr-qc].

[29] A. Einstein, Sitzungsber. Preuss. Akad. Wiss. Berlin (Math. Phys. ) 1917, 142 (1917)

[30] E.A. Ivanov, Phys. Part. Nucl. 47, no. 4, 508 (2016)

[31] S. Chandrasekhar, The mathematical theory of black holes (Oxford Clarendon, UK, 1985) 1.

[32] C.W. Misner, K.S. Thorne and J.A. Wheeler, Gravitation (San Francisco, USA, 1973) 
[33] C. Kiefer, Int. Ser. Monogr. Phys. 124, 1 (2004)

[34] B.M. Barbashov, V.N. Pervushin, A.F. Zakharov and V.A. Zinchuk, Phys. Lett. B 633, 458 (2006)

[35] A. Lichnerowicz, J. Math. Pures. Appl. 23, 37 (1944)

[36] J.W. York, Jr., Phys. Rev. Lett. 26, 1656 (1971) 